\title{
Chronic Inflammatory Demyelinating Polyneuropathy (CIDP) and Dilated Cardiomyopathy: A New Association?
}

\author{
Magro VM${ }^{1 *}$, Verrusio $\mathrm{W}^{2}$ and Lama $\mathrm{D}^{1}$ \\ ${ }^{1}$ Department of Internal Medicine and Geriatry, University of Campania “Luigi Vanvitelli", Italy \\ ${ }^{2}$ Department of Cardiovascular, Respiratory, Nephrological, Anesthesiological and Geriatric Sciences, Sapienza University of Rome, Italy
}

Submission: September 08, 2018; Published: October 09, 2018

*Corresponding author: Valerio Massimo Magro, Department of Internal Medicine and Geriatry, University of Campania "Luigi Vanvitelli", Piazza

L. Miraglia 2, 80100, Naples, Italy, Tel: (+39) 3492224922; Email: valerio_magro@hotmail.com

\section{Abstract}

Chronic Inflammatory Demyelinating Polyneuropathy (CIPD) belongs to the most common immune-mediated chronic disorders of the peripheral nervous system and intravenous immunoglobulin is considered the first-line treatment. in addition to being characterized by numerous phenotypic variants, this condition has been described as associated with other pathologies that have an immune pathogenesis. We describe the clinical case of a patient in whom this condition we discovered was associated with the presence of dilated cardiomyopathy. We tried to be learned from this clinical case report the implications, as well as clinics, even pathogenetic both on the CIDP pathogenesis and on cell-mediated and humoral mechanisms act synergistically to cause damage to cardiac muscle, to be able to derive a therapeutic approach that was optimal.

Keywords: Chronic inflammatory demyelinating polyradiculoneuropathy; Immunoglobulins; Heart failure with low ejection fraction; Dilated cardiomyopathy; Echocardiogram

Abbreviations: CIPD: Chronic Inflammatory Demyelinating Polyneuropathy; VEBs: Ventricular Ectopic Beats; EF: Ejection Fraction; LA: Left Atrium; NTproBNP: N-terminal pro-brain natriuretic peptide; SCIg: Subcutaneous Ig

\section{Introduction}

Chronic Inflammatory Demyelinating Polyradiculoneuropathy (CIDP) is an inflammatory neuropathy, characterised by a slowly progressive onset and symmetrical, sensorimotor involvement, and many phenotypic variants which have the common denominator a prognosis characterized by disability $[1,2]$. The therapy of this condition makes use of immunomodulating drugs: corticosteroids, immunoglobulins, azathioprine and other immunosuppressive agents [3]. This condition often does not exist by itself, but it is associated with other diseases, one of which we describe.

\section{Materials and Methods}

We evaluated a patient who had a diagnosis of CIPD. A 52-yearold man presented gradual limb weakness, predominantly in lower limbs, with associated body weight loss observed by about 2 months and moderate fatigue. The patient's medical records showed hypothyroidism replacement therapy (thyroxine $50 \mathrm{mcg}$ ) and previous diagnosis of CIPD treated with steroids and immunoglobulins (first intravenously and subcutaneously currently). The subject appeared well oriented and without cognitive impairment (Glasgow Coma Score - GCS - 15/15; Mini
Mental State Examination - MMSE - 30/30), vital signs within the limits but signs of myogenic and neurogenic suffering (physical examination showed the presence of superficial and deep hypostenia, hypotrophy and hypoesthesia, more distal to proximal, with osteotendinous areflexia) and low body mass index (BMI $\left.19 \mathrm{~kg} / \mathrm{m}^{2}\right)$.

\section{Results}

After examining the subject clinically and through dedicated scales, we proceeded to a patient evaluation both from the hematochemical and instrumental point of view. Blood chemistry analysis showed normal count of White Blood Cells (WBC) $(6,22$ cells/mcL; n.v. 4,8 to 10,8 cells/mcL; leucocyte formula within the limits), normoalbuminemia $(4.0 \mathrm{~g} / \mathrm{dl}$, n.v. $3.5-5.5 \mathrm{~g} / \mathrm{dl})$, euglycaemia (81 mg/dl, n.v. 70-100), normal renal (creatinine 0,71 mg/dl, n.v. 0.51-0.95), hepatic (AST 24 U/l, n.v. 5-32; ALT $11 \mathrm{U} / \mathrm{l}$, n.v. 5-33; total bilirubin $0.25 \mathrm{mg} / \mathrm{dl}$, n.v. $<1,2$ ) and thyroid function; the chronic inflammatory marker were increased (ESR $51 \mathrm{~mm} / \mathrm{h}$; n.v. 0-35 mm/h) but not the acute marker (CRP 0.09 $\mathrm{mg} /$ dl; n.v. 0-0,5). Urinalysis, electrolytes and markers of hepatitis showed no significant abnormalities. The electroneurography 


\section{Journal of Cardiology \& Cardiovascular Therapy}

showed widespread damage of peripheral nerve fibers (Figure 1). Electrocardiogram there was a sinus rhythm with 75 heart beats per minute and the presence of some Ventricular Ectopic Beats (VEBs), without significant asystolic pause, confirmed in 24 hours-cardiac monitoring (Figure 2). Then we performed an echocardiogram (exclusion of organic arrhythmogenic focus or myocarditis) finding a larger size of left ventricular with hypoakinesia of SIV, which appeared normal thickness. Ejection Fraction (EF) about 40\%. Mitral with sclerotic edges. Left Atrium (LA) within normal limits (Vol LA/BSA $13.7 \mathrm{ml} / \mathrm{mq}$ ). Aortic root within the limits. Right sections within the limits (right atrial area $11 \mathrm{~cm}^{2}$ ). At Doppler, there was low grade mitral and tricuspid regurgitation. PAPs $30 \mathrm{~mm} \mathrm{Hg}$. Reversed transmitral pattern as impaired diastolic relaxation (Figure 3). While the subsequent myocardial scintigraphy proved negative for ischemia induced by stress, cardiac magnetic resonance imaging did not appreciate areas of altered signal (the search for edema/ myocardial inflammation in STIR sequences was negative) but confirmed the finding left ventricular dilation and decreased ejection fraction (39\%), confirming the diagnosis of idiopathic dilated cardiomyopathy. N-terminal pro-brain natriuretic peptide (NTproBNP) was $300 \mathrm{pg} / \mathrm{ml}$. Detecting anti beta1-adrenergic receptor antibodies was not performed for non-availability of the laboratory to perform this dosage.

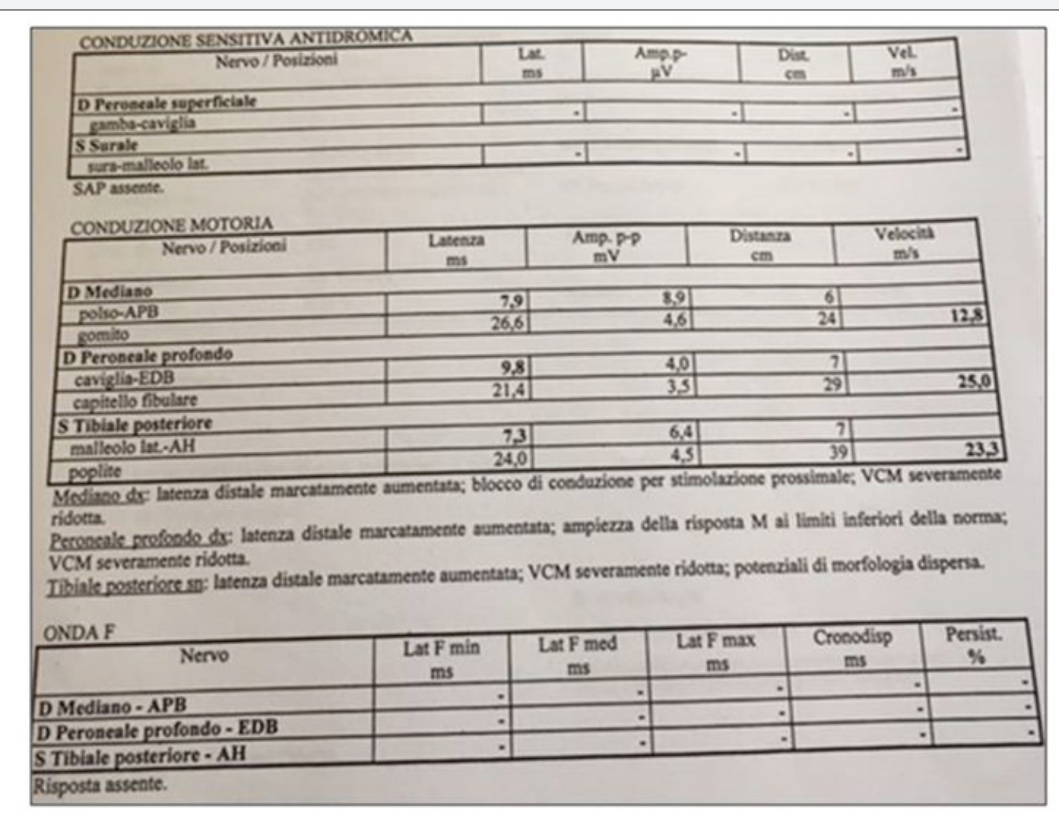

\section{ELECTRONEUROGRAPHY}

Figure 1: Electroneurography. The exam shows sensitives the absence of the Sensitives Action Potentials (SAP) at the level of the superficial peroneal nerve and the sural nerve and a motor conduction characterized by a distal latency markedly increased in the right median nerve, in the right deep peroneal nerve and in the rear left tibial nerve.

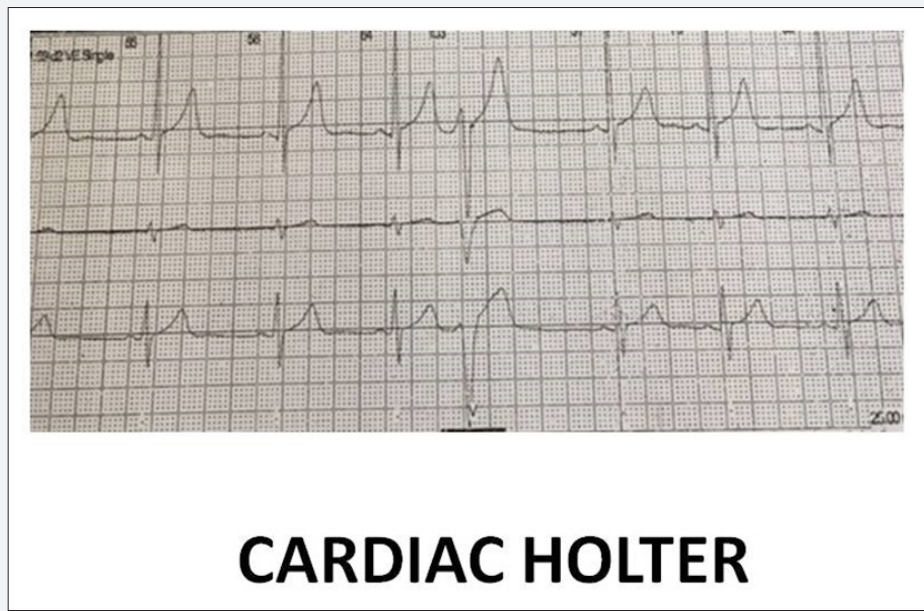

Figure 2: 24-hour Holter electrocardiography. The exam shows sinus rhythm with a mean heart rate 74 beats and sinus arrhythmia phases during the night. During the monitoring we observed 64 isolated bimorph ventricular ectopic beats (VEBs) and 4 supraventricular ectopic beats (SVEBs). Pauses $>2.5$ seconds were absent. 

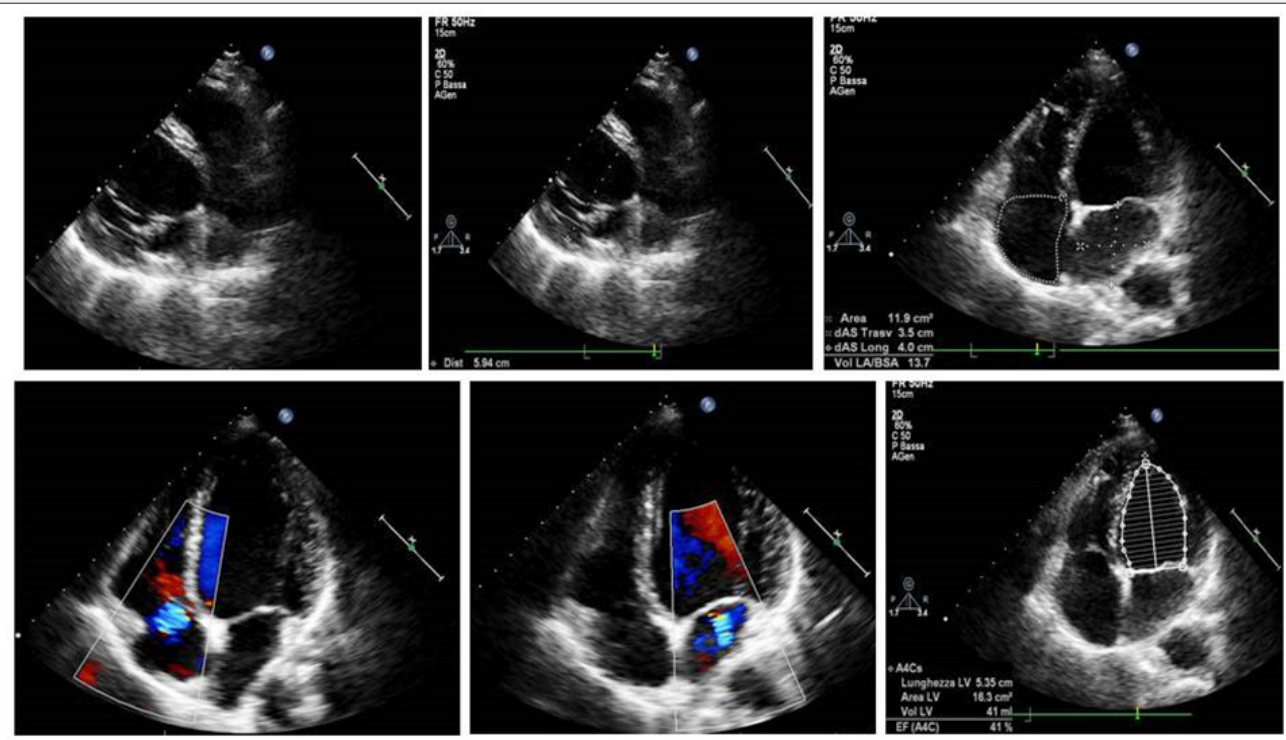

\section{ECHOCARDIOGRAPHY}

Figure 3: Echocardiogram. The examination shows a dilation of the left ventricle with a significant systolic dysfunction. These findings were consistent with the subsequent examination of cardiac magnetic resonance imaging and, together with myocardial scintigraphy, they confirmed the diagnosis of idiopathic dilated cardiomyopathy.

The patient was treated during hospitalization with intravenous injections of methylprednisolone sodium hemisuccinate $(1$ g) and a self-infused subcutaneous Ig (SCIg) G therapy (Hizentra®). Then, he was discharged with the following therapy: thyroxine $50 \mathrm{mcg}$, betamethasone phosphate $4 \mathrm{mg}$ for three days and to follow $1.5 \mathrm{mg}$ sine die, Hizentra ${ }^{\circledR}$, metoprolol $100 \mathrm{mg}$, alpha lipoic acid $600 \mathrm{mg}$, with an improvement in symptoms and general conditions at the follow-up three months later, a recovery of body weight (increase of $3 \mathrm{~kg}$ ) and a slight decrease in the NTproBNP levels $(280 \mathrm{pg} / \mathrm{ml})$.

\section{Discussion}

In addition to the wide range of CIDP phenotypes, several related immune-mediated disorders have been described. In fact, in literature were observed associations with various diseases, quite heterogeneous for clinical manifestations and affected organs among themselves, such as amyotrophic lateral sclerosis [4], Crohn's disease [5], diabetes [6], glomerulonephritis [7], infectious pathologies [8] and hemopathies [9] and other conditions yet. There are no described cases of associations with cardiovascular disease and demyelinating polyradiculoneuropathy and this patient, to our knowledge, is the first reported case of Dilated Cardiomyopathy (DCM) combined with CIDP. In the course of our investigations we excluded both ischemic that iatrogenic events (immunoglobulins; however, these adverse events have never been described in patients treated with the used product, Hizentra ${ }^{\circledR}$ ). The pathogenesis of DCM remains in large part not well known yet, but several studies have provided evidence that autoimmunity is one of the pivotal mechanisms $[10,11]$. Unfortunately, it was not possible to measure the antibodies against the beta1-adrenergic receptor and our treatment consisted of agents who acted on cardiac remodeling, such as beta blockers and alpha lipoic acid [12,13]. Therefore, Igs are believed to play an important role in the myocardial damage at the base of the pathology and the removal of specific Ig may be an effective treatment of patients with secondary heart failure, as in our patient, without compromising the CIPD therapy $[14,15]$.

\section{Conclusion}

CIDP is an autoimmune disorder with progressive weakness and also DCM, with the consequent heart failure, can manifest clinically with a generalized asthenia that, with the concomitance of the two conditions, can be particularly severe and undermine the quality of life of the patient. The knowledge of the pathogenetic mechanisms of these pathologies, especially where there may be common pathways, can be decisive for an optimal therapy and to significantly attenuate the clinical manifestations of both diseases.

\section{Conflict of interest}

All authors declare no conflict of interest.

\section{References}

1. Mathey EK, Park SB, Hughes RA, Pollard JD, Armati PJ, et al. (2015) Chronic inflammatory demyelinating polyradiculoneuropathy: from pathology to phenotype. J Neurol Neurosurg Psychiatry 86(9): 973985.

2. Reynolds J, Sachs G, Stavros K (2016) Chronic Inflammatory Demyelinating Polyradiculoneuropathy (CIDP): Clinical Features, Diagnosis, and Current Treatment Strategies. R I Med J (2013) 99(12): $32-35$

3. Oaklander AL, Lunn MP, Hughes RA, van Schaik IN, Frost C, et al. (2017) Treatments for chronic inflammatory demyelinating polyradiculoneuropathy (CIDP): an overview of systematic reviews. Cochrane Database Syst Rev 1: CD010369. 
4. Akaishi T, Tateyama M, Kato K, Miura E, Izumi R, et al. (2014) An autopsy case involving a 12-year history of amyotrophic lateral sclerosis with CIDP-like polyneuropathy. Intern Med 53(12): 1371-1375.

5. Kim S, Kang SJ, Oh KW, Ahn BK, Lee HL, et al. (2015) Chronic inflammatory demyelinating polyneuropathy-like neuropathy as an initial presentation of Crohn's disease. BMC Neurol 15: 48.

6. Bril V, Blanchette CM, Noone JM, Runken MC, Gelinas D, et al. (2016) The dilemma of diabetes in chronic inflammatory demyelinating polyneuropathy. J Diabetes Complications 30(7): 1401-1407.

7. Sung WK, Jeong JU, Bang KT, Shin JH, Yoo JH et al. (2015) Fibrillary glomerulonephritis combined with chronic inflammatory demyelinating polyneuropathy. Kidney Res Clin Pract 34(2): 117-119.

8. Lim JY, Lim YH, Choi EH (2016) Acute-onset chronic inflammatory demyelinating polyneuropathy in hantavirus and hepatitis $\mathrm{B}$ virus coinfection. Medicine (Baltimore) 95(49): e5580.

9. Jaafoura NG, Atig A, Bouker A, Alaoua O, Khalifa M, et al. (2014) Chronic inflammatory demyelinating polyradiculoneuropathy revealing essential thrombocythemia. Pan Afr Med J 19: 101.

10. Fu M (2008) Autoimmunity and idiopathic dilated cardiomyopathy: where we stand? Autoimmunity 41(6): 415-418.
11. Arndt-Marić R, Nägele H, Schewe G, Kromminga A (2010) Are autoantibodies against the beta1-adrenergic receptor markers for dilated cardiomyopathy? Clin Lab 56(11-12): 519-526.

12. Marfella R, Barbieri M, Sardu C, Rizzo MR, Siniscalchi M, et al. (2016) Effects of $\alpha$-lipoic acid therapy on sympathetic heart innervation in patients with previous experience of transient takotsubo cardiomyopathy. J Cardiol 67(2): 153-161.

13. Hegazy SK, Tolba OA, Mostafa TM, Eid MA, El-Afify DR, et al. (2013) Alpha-lipoic acid improves subclinical left ventricular dysfunction in asymptomatic patients with type 1 diabetes. Rev Diabet Stud 10(1): 58-67.

14. Patel PA, Hernandez AF (2013) Targeting anti-beta-1-adrenergic receptor antibodies for dilated cardiomyopathy. Eur J Heart Fail 15(7): 724-729.

15. Yoshikawa T, Baba A, Akaishi M, et al. (2016) Immunoadsorption therapy for dilated cardiomyopathy using tryptophan column -A prospective, multicenter, randomized, within-patient and parallelgroup comparative study to evaluate efficacy and safety. J Clin Apher 31(6): 535-544.

\section{Your next submission with Juniper Publishers} will reach you the below assets

- Quality Editorial service

- Swift Peer Review

- Reprints availability

- E-prints Service

- Manuscript Podcast for convenient understanding

- Global attainment for your research

- Manuscript accessibility in different formats

( Pdf, E-pub, Full Text, Audio)

- Unceasing customer service

Track the below URL for one-step submission https://juniperpublishers.com/online-submission.php 